\title{
A Tunable $S$-Band Erbium-Doped Fiber Ring Laser
}

\author{
Chien-Hung Yeh, Chien-Chung Lee, and Sien Chi
}

\begin{abstract}
In this paper, we experimentally investigate and demonstrate an $S$-band erbium-doped fiber (EDF) ring laser based on an erbium-doped silica fiber and 980-nm pumping laser. Widely tunable range from 1480 to $1522 \mathrm{~nm}$, and the sidemode suppression ratio (SMSR) of $>30 \mathrm{~dB} / 0.1 \mathrm{~nm}$ and the output power of $>-2 \mathrm{dBm}$ in the operating range from $1482 \mathrm{~nm}$ to $1517 \mathrm{~nm}$ have been achieved for this ring laser. Under the constant power control, the output power variation less than $\pm 0.05 \mathrm{~dB}$ can also be accomplished over the tuning range from 1482 to $1517 \mathrm{~nm}$. This $S$-band EDF ring laser is promising for the future $S$-band applications.
\end{abstract}

Index Terms-EDF, fiber ring laser, $S$-band.

\section{INTRODUCTION}

$\mathbf{E}$ RBIUM-DOPED FIBER (EDF) ring lasers are applicable to loss measurements of optical components, optical sensing systems, and tunable transmitters in wavelength-division-multiplexing (WDM) systems. Due to the bandwidth limitation of erbium-doped fiber amplifiers (EDFAs), the wavelength range of erbium-doped fiber ring lasers can only cover both $C$ - and $L$-bands (1530-1610 nm) [1]-[3]. Recently, an $S$-band (1450-1530 nm) amplification technique, which utilizes erbium-doped silica fiber with depressed cladding design and 980-nm pump laser to generate EDF gain extension effect, has been reported [4]. By using this $S$-band amplifier, the operating wavelengths of EDF ring lasers can be extended to $S$-band range.

In this letter, we have experimentally investigated and demonstrated the feasibility of a tunable $S$-band ring laser by using the similar configuration for $C$ - and $L$-band ring lasers. The performances of tunable range, output power, sidemode suppression ration (SMSR), and constant gain control have also been studied.

\section{EXPERIMENTS AND DISCUSSIONS}

Fig. 1 shows the experimental setup for the tunable $S$-band EDF ring laser. This configuration was constructed by a $1 \times 2$ and 10:90 optical coupler, an polarization controller (PC), an intracavity fiber Fabry-Pérot filter (FFP-TP), and an $S$-band EDFA module composed of two stage EDFA and a power-sharing 980 -nm pump laser. The $S$-band EDF inside EDFA module has a depressed cladding design in order to

Manuscript received March 17, 2003; revised April 30, 2003. This work was supported in part by the Academic Excellence Program of R.O.C. Ministry of Education under Contract 89-E-FA06-1-4-90X023, and the National Science Council of R.O.C. under Contract NSC-91-2215-E-009-027.

The authors are with the Institute of Electro-Optical Engineering, National Chiao-Tung University, Hsinchu 30050, Taiwan, R.O.C. (e-mail: depew.eo89g@nctu.edu.tw).

Digital Object Identifier 10.1109/LPT.2003.815314

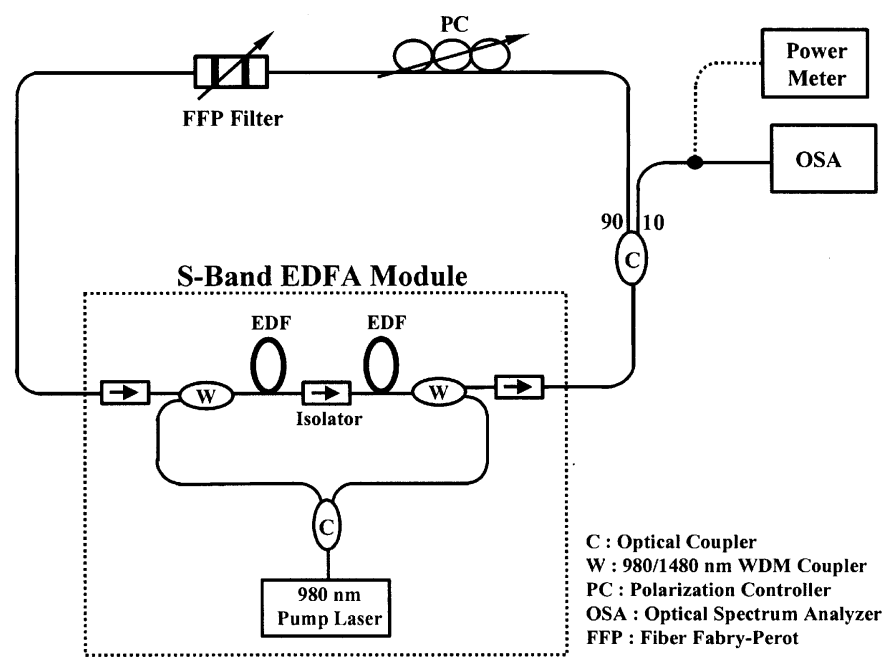

Fig. 1. The experimental setup for an $S$-band EDF ring laser.

provide a sharp, high-attenuation, long-wavelength cutoff filter into active fibers. The erbium-doped fibers in the first and second stages have different characteristics. The fiber in the first stage has the fiber length of $20 \mathrm{~m}$, and can provide low noise figure and medium gain by forward pumping. The fiber in the second stage has the fiber length of $30 \mathrm{~m}$, and can produce large output power by backward pumping. In addition, the optical isolator between these two stages can reduce backward amplified spontaneous emission (ASE) and improve noise figure performance. The gain and noise figure can reach $32 \mathrm{~dB}$ and $5.7 \mathrm{~dB}$ at $1500 \mathrm{~nm}$ for input power of $-25 \mathrm{dBm}$, and the saturated output power at $1500 \mathrm{~nm}$ can be up to $14 \mathrm{dBm}$ for input power of $0 \mathrm{dBm}$. The total pump power of this amplifier module can be up to $280 \mathrm{~mW}$ while the bias current is operated at $356 \mathrm{~mA}$. The FFP filter is an all-fiber device having a widely tunable range, low insertion loss $(<0.5 \mathrm{~dB})$, and low polarization-dependent loss $(\sim 0.1 \mathrm{~dB})$. This FFP filter having the free spectral range (FSR) of $44.5 \mathrm{~nm}$ and the finesse of $200 \mathrm{~nm}$ can provide wavelength selection in the ring laser cavity by applying external voltage $(<12 \mathrm{~V})$ on the piezoelectric transducer (PZT) of FFP filter. In addition, an optical spectrum analyzer (OSA) and a power meter (PM) are used to measure the output spectra and powers of the ring laser.

Fig. 2 shows the optical spectra of this EDF ring laser while the various external-voltage applied on the PZT of FFP filter. The lasing wavelength tuning over $42 \mathrm{~nm}$ from 1480 to $1522 \mathrm{~nm}$ is observed. The ring laser has ASE and high output power at the high gain region near $1508 \mathrm{~nm}$. Furthermore, the insert of Fig. 2 is the ASE spectrum of the $S$-band amplifier. Fig. 3 represents the output power and SMSR versus the tuning wavelength for this ring laser. The maximal output power of $5.1 \mathrm{dBm}$ occurs at around $1498 \mathrm{~nm}$, and the output powers drop to $-3.0,-6.0$, 


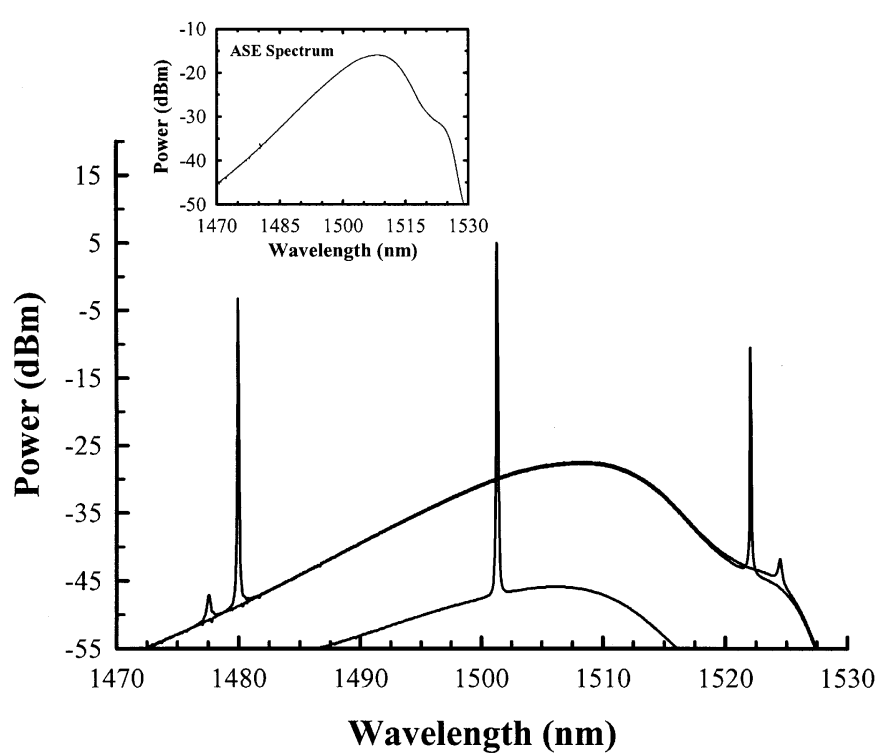

Fig. 2. The optical spectra of tuning wavelength in the EDF fiber ring laser when the external-voltage applied on the PZT of FFP filter over a tuning range from 1480 to $1522 \mathrm{~nm}$. The insert is the ASE spectrum of an $S$-band EDFA module.

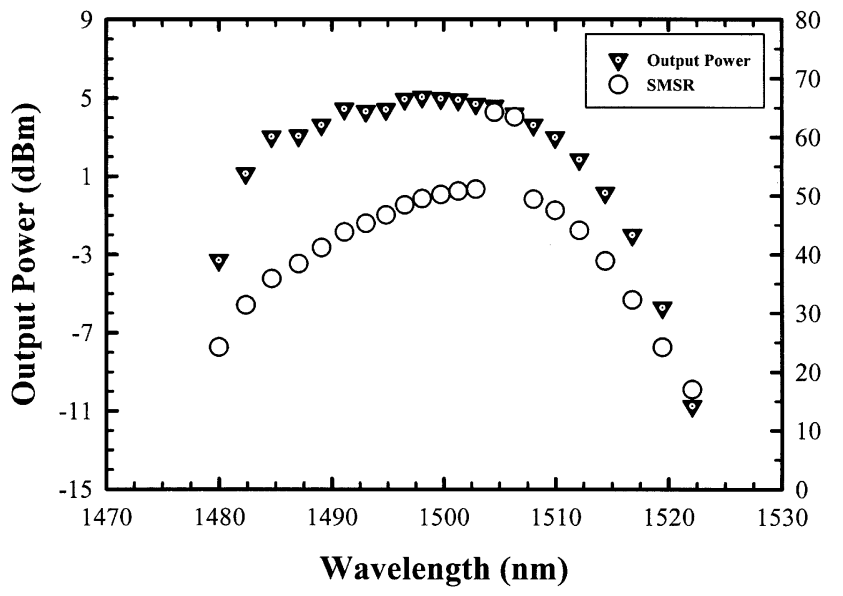

Fig. 3. The output power and SMSR versus the tuning wavelength for this ring laser over the tunable range from 1480 to $1522 \mathrm{~nm}$.

and $-11.0 \mathrm{dBm}$ while operating at 1480,1520 and $1522 \mathrm{~nm}$, respectively. As seen in Fig. 3, the SMSR and output power can be kept larger than $30 \mathrm{~dB} / 0.1 \mathrm{~nm}$ and $-2.0 \mathrm{dBm}$ in a wide tuning range over $34 \mathrm{~nm}(1482-1517 \mathrm{~nm})$. When the lasing wavelength operated at around $1508 \mathrm{~nm}$, the SMSR will increase dramatically to $64.2 \mathrm{~dB} / 0.1 \mathrm{~nm}$ due to the strong gain competition and ASE compression. As a result, the tunable range over $42 \mathrm{~nm}(1480-1522 \mathrm{~nm})$ can be achieved and the SMSR of $>30 \mathrm{~dB} / 0.1 \mathrm{~nm}$ and the output power of $>-2 \mathrm{dBm}$ can be kept in the operating region from 1482 to $1517 \mathrm{~nm}$ for this EDF ring laser.

Furthermore, the constant output power control for this ring laser can be obtained by proper adjustment of the bias current of the pump laser in the $S$-band amplifier. Fig. 4 indicates the

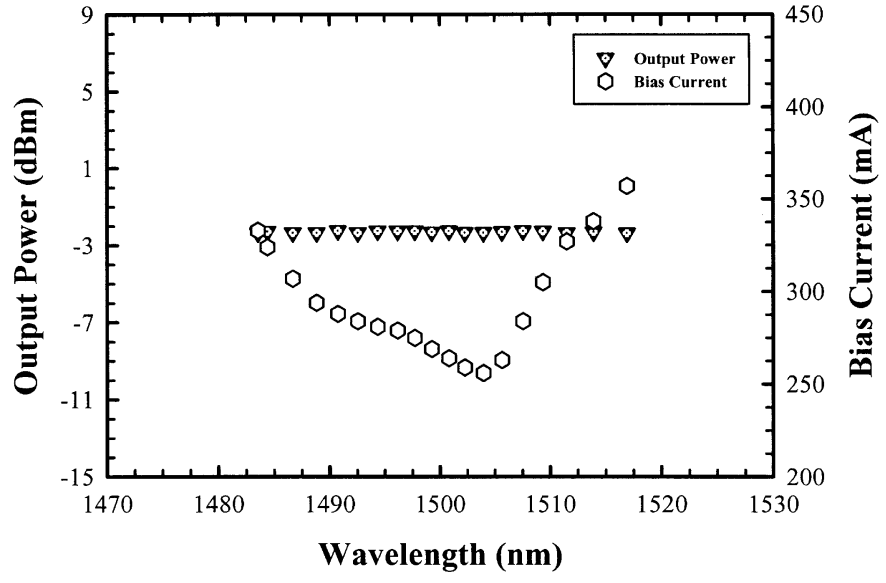

Fig. 4. The constant output power (set at $-2.3 \mathrm{dBm}$ ) and various bias current $(255-356 \mathrm{~mA})$ of the pump laser versus lasing wavelength for this ring laser in the tuning range from 1482 to $1517 \mathrm{~nm}$.

output power and bias current of the pump laser versus lasing wavelength for this ring laser. In Fig. 4, we set the constant output power of this ring laser at $-2.3 \mathrm{dBm}$. The power variation less than $\pm 0.05 \mathrm{~dB}$ can be obtained in the tuning range from 1482 to $1517 \mathrm{~nm}$ by tuning the bias current from 255 to $356 \mathrm{~mA}$.

\section{CONCLUSION}

In summary, an $S$-band EDF ring laser based on an erbiumdoped silica fiber and 980-nm pumping laser have been experimentally investigated and demonstrated. Widely tunable range from 1480 to $1522 \mathrm{~nm}$, and the SMSR of $>30 \mathrm{~dB} / 0.1 \mathrm{~nm}$ and the output power of $>-2 \mathrm{dBm}$ in the operating range from 1482 to $1517 \mathrm{~nm}$ have been achieved for this ring laser. Under the constant power control, the output power variation less than $\pm 0.05 \mathrm{~dB}$ can also be accomplished over the tuning range from $1482 \mathrm{~nm}$ to $1517 \mathrm{~nm}$. This $S$-band EDF ring laser is promising for the future $S$-band applications.

\section{ACKNOWLEDGMENT}

Authors would like to thank C.-Y. Chen for help on experiments.

\section{REFERENCES}

[1] Y. Sun, J. W. Sulhoff, A. K. Srivasta, J. L. Zyskind, T. A. Strasser, J. R. Pedrazzani, C. Wolf, J. Zhou, J. B. Judkins, R. P. Espindola, and A. M. Vengsarkar, " $80 \mathrm{~nm}$ ultra-wideband erbium-doped silica fiber amplifier," Electron. Lett., vol. 33, no. 23, pp. 1965-1967, 1997.

[2] L. Talaverano, S. Abad, S. Jarabo, and M. Lopez-Amo, "Multiwavelength fiber laser sources with Bragg-grating sensor multplexing capability," J. Lightwave Technol., vol. 19, pp. 553-558, Apr. 2001.

[3] S. Yamashita and M. Nishihara, "Widely tunable erbium-doped fiber ring laser covering both C-band and L-band," IEEE J. Select Topics Quantum Electron, vol. 7, pp. 41-43, Jan./Feb. 2001.

[4] M. A. Arbore, Y. Zhou, G. Keaton, and T. Kane, "34 dB gain at 1500 $\mathrm{nm}$ in S-band EDFA with distributed ASE suppression," in 28th ECOC 2002, vol. 1, 2002. 